\title{
Tiefes chemisches Peeling mit Phenol - Renaissance eines Goldstandards?
}

\section{Deep Chemical Peeling with Phenole - Renaissance of a Gold Standard}

Autoren

Institute
T. Walker ${ }^{1}$, L. Wiest ${ }^{2}$, A. Boer ${ }^{3}$, C. Bayerl ${ }^{1}$

Klinik für Dermatologie und Allergologie, Dr. Horst Schmidt Kliniken, Wiesbaden

2 Hautärztin, Residenzstraße 7, München

Dermatologikum Hamburg
Bibliografie

DOI http://dx.doi.org/ 10.1055/s-0029-1215077

Online-Publikation: 28. 9. 2009

Akt Dermatol 2010; 36:

73-82 @ Georg Thieme

Verlag KG Stuttgart · New York ISSN 0340-2541

Korrespondenzadresse Dr. med. Torsten Walker Klinik für Dermatolgie und Allergologie HSK, Wilhelm Fresenius Klinik Städtische Klinikum Wiesbaden Lehrkrankenhaus der Universität Mainz Aukammallee 39 65191 Wiesbaden Torsten_Walker@gmx.de

\section{Zusammenfassung $\nabla$}

Chemische Peelingverfahren zur Hautverjüngung haben in der Geschichte der Medizin eine lange Tradition. Bereits im Papyrus Ebers wurden vor 3500 Jahren erste chemische Peels beschrieben. Das Phenol-Peeling wurde in den 40er-Jahren des letzten Jahrhunderts von Laienoperateuren in den USA durchgeführt. Baker und Gordon modifizierten die Lösung und der wissenschaftliche Durchbruch mit Anerkennung der Methode erfolgte in den 60er-Jahren. Seither ist es eine Standardmethode der Hautverjüngung. Mit Einführung der Lasertechnologie wurde das PhenolPeeling zurückgedrängt. Mit einiger Erfahrung

\section{Allgemeine Überlegungen \\ $\nabla$}

Die Maßnahmen zur Hautverjüngung sind zahlreich und aus dem Repertoire eines ästhetisch arbeitenden Dermatologen nicht mehr wegzudenken. Die Anwendungen von Botulinumtoxin A und Fillern sollte beherrscht werden. Sie haben in der kosmetischen Dermatologie ihren berechtigten Stellenwert. Allerdings kann nicht jede Falte mit Augmentationsverfahren befriedigend verbessert werden. Neben der Faltenausbildung lassen weitere „Komponenten“ unsere Haut alt erscheinen. Zum einen kommt es zur Ausbildung oder Weitstellung von Gefäßen, das heißt vaskuläre Prozesse spielen eine Rolle. Aber auch fahles Hautkolorit und Grobporigkeit sind neben Pigmentierungen Kennzeichen der alternden Haut. Die jugendlich wirkende Haut zeichnet sich neben ihrer Faltenlosigkeit auch durch einen helleren und klaren Teint sowie durch Feinporigkeit aus. Die älter werdende Haut bekommt einen mehr gräulich-bräunlichen Farbton. Hier besitzt das chemische Peeling mit Phenol einen unschlagbaren Vorteil gegenüber dem Face-lift, da es sowohl Hauttextur und Struktur, Pigmentie- nach $\mathrm{CO}_{2}$-Laser skin resurfacing wurde allerdings auch diese Methode wegen der Nebenwirkungen und der teils unbefriedigenden kosmetischen $\mathrm{Er}$ gebnisse wieder verlassen. Einige neue Lasersysteme sind zurzeit auf dem Markt, müssen sich aber erst bewähren und ihr Stellenwert wird sich erst in einigen Jahren zeigen. Das tiefe Peeling mit Phenol bietet sehr gute und verlässliche Ergebnisse, die darüber hinaus lang anhaltend sind. Es handelt sich hierbei aber um eine dermatologische, invasive Maßnahme, die Erfahrung und einer intensiven Patientenführung bedarf. Die kosmetischen Ergebnisse sind überzeugend und führen zu einer hohen Patientenzufriedenheit.

rungen und das Hautkolorit positiv beeinflusst. Dadurch tritt - auch wenn der Straffungseffekt geringer als beim Face-lift ist - ein nicht zu unterschätzender Verjüngungseffekt mit der Anwendung eines einzigen Verfahrens ein.

\section{Geschichte des chemischen Peels \\ $\nabla$}

Die Ägypter der Antike badeten die Haut in Eselsmilch zur Verbesserung der Elastizität, wie im Papyrus Ebers vor 3500 Jahren beschrieben. Sicherlich war das Wissen, dass der hautverjüngende Effekt auf der $\alpha$-Hydroxy-Milchsäure beruht, unbekannt. 1882 entdeckte der deutsche Dermatologe Unna die Wirkung von Resorchin, Salicylsäure, Phenol und Trichloressigsäure an der Haut und beschrieb sie [1]. Der britische Dermatologe Mackee benutzte bereits 1903 Phenol-Lösung zur Aknetherapie und publizierte seine Ergebnisse 1952 [2]. Während des Ersten Weltkrieges wurde Phenol-Lösung von Dr. La Gassé zur Therapie von Pulvereinsprengungen im Gesichtsbereich mit guten kosmetischen Ergebnissen eingesetzt. Hierbei wurde bereits ein Okklusivverband nach Ap- 


\begin{tabular}{ll}
\hline $\begin{array}{l}\text { Tab. } 1 \text { Indikationen für chemisches Peeling. } \\
\text { oberflächiges Peeling } \\
\text { (AHA, Salizylsäure, }\end{array}$ & $\begin{array}{l}\text { ergänzende Therapie bei Akne comedonica, } \\
\text { papulo-pustulosa, Verhornungsstörungen, } \\
\text { epidermale Pigmentläsionen, Hautalterung, } \\
\text { TCA 30\%) }\end{array}$ \\
\hline $\begin{array}{l}\text { Grobporigkeit } \\
\text { (TCA 35-50\%) }\end{array}$ & $\begin{array}{l}\text { moderate Falten, Photoaging, Lentigines } \\
\text { solares, Hyperpigmentierungen, oberfläch- } \\
\text { liche Narben }\end{array}$ \\
\hline $\begin{array}{l}\text { tiefes Peeling } \\
\text { (Phenol) }\end{array}$ & $\begin{array}{l}\text { tiefe Falten, ausgeprägte Aknenarben, } \\
\text { ausgeprägtes Fotoaging }\end{array}$ \\
\hline
\end{tabular}

plikation des Phenols angewendet. Seine Tochter Antoinette verwendete dieses Verfahren 1930 und 1940 in der Nähe von Los Angeles zur Faltenbehandlung. Diese Laienoperateure richteten das „House of Renaissance“ ein, in dem u.a. auch Silikoninjektionen erfolgten. Eines dieser Häuser war dann auch in Florida beheimatet. Das Ehepaar Adolph und Marthe Brown, er Mund-Kiefer- und Gesichts-Chirurg und sie Dermatologin, führten in den späten 50er- und frühen 60er-Jahren histologische Studien zum Phenol-Peel durch. Zu diesem Zeitpunkt waren unterschiedliche Phenol-Mischungen auf dem Markt, die neben Phenol u.a. Salicylsäure, Oliven- oder Sesamöl enthielten. Zu diesem Zeitpunkt erfuhr der plastische Chirurg Dr. Thomas Baker in Miami, dass es in Los Angeles und auch in Miami eine „mysteriöse Lösung“ zur Faltenbehandlung gäbe. Laienoperateure arbeiteten bislang mehr oder weniger im Verborgenen und die genaue Zusammensetzung der Lösung war geheim. In Zusammenarbeit mit dem Ehepaar Brown und Gordon wurden dann auf einem Kongress von Baker ausgezeichnete fotografisch dokumentierte Ergebnisse präsentiert, die zur fachlichen Anerkennung der Baker-GordonPhenol-Peels führten $[3,4]$. In den 70er-Jahren war das Chemical Peeling ein häufig durchgeführtes Verfahren zur Hautverjüngung und zahlreiche Ergebnisse und Studien wurden publiziert. Weitere Säuren wie Trichloressigsäure oder $\alpha$-Hydroxysäuren (Fruchtsäuren) etablierten sich. Die Arbeit von Stegman in den 80ern am Tiermodell und menschlichen Modell verglich die histologischen Effekte von Peeling und Dermabrasion und war wegbereitend für die Durchführung des chemischen Peelings (Rezeptur siehe unten) $[5,6]$.

\section{Allgemeine Klassifikation der chemischen Peels \\ $\nabla$}

Je nach Art der verwendeten Substanz können unterschiedliche Eindringtiefen mit unterschiedlichen Ergebnissen erreicht werden. Dieses ist entscheidend für die Indikationsstellung (๑ Tab. 1).

\section{Oberflächiges Peeling}

Hier haben sich die Fruchtsäuren ( $\alpha$-Hydroxysäuren), insbesondere die Glykolsäure durchgesetzt. Diese sind kommerziell erhältlich und relativ einfach in der Handhabung. Meist können diese Peelings von fachlich geschultem Personal unter ärztlicher Aufsicht durchgeführt werden. Indikationen sind Akne vulgaris, allerdings nicht als alleinige Therapieform, sondern am besten in Kombination mit einer differenzierten Lokaltherapie. Des Weiteren kann eine Verbesserung der Hautstruktur und Textur erreicht werden. Antikomedogene und antientzündliche Eigenschaften besitzt die Fruchtsäure nicht, ihre Wirkweise beruht auf einer pH-Verschiebung ins saure Milieu. Der Effekt der Fruchtsäure ist limitiert; die Eindringtiefe rein epidermal, ca.
0,06 mm. Durch Kombination mit Salicylsäure kann die Wirkweise verstärkt werden. Zur Faltenbehandlung eignet sich die Fruchtsäure nicht. Indikation siehe Tab. 1. Weitere oberflächliche Peeling-Substanzen sind: Salicylsäure, Pyruvatsäure, Jessner'sche Lösung und Trichloressigsäure bis zu einer Konzentration von $30 \%$. Aber auch Adapalen und Azelainsäure sind oberflächlich schälende Substanzen.

\section{Mitteltiefes Peeling}

Hier ist die Eindringtiefe ca. $0,45 \mathrm{~mm}$ bis in die papilläre Dermis. Dieses kann mit Trichloressigsäure in einer Konzentration von $60 \%$ erreicht werden. Allerdings ist die Säure nicht kontrollierbar, das Narbenrisiko hoch. Daher ist man dazu übergegangen, die Konzentration der Trichloressigsäure nicht höher als 35\%ig zu wählen und sie dafür zusätzlich mit anderen Säuren zu kombinieren, um eine entsprechende Eindringtiefe zu erreichen, die bis in die papilläre Dermis reicht. Hierfür stehen unterschiedliche Kombinationsmöglichkeiten zur Verfügung.

1. Nach Brody: $\mathrm{CO}_{2}$ und TCA $35 \%$

2. Nach Coleman: Glykolsäure und TCA $35 \%$

3. Nach Monheit: Jessner'sche Lösung und TCA 35\%

4. Nach Grimes: Salicylsäure 10\% und TCA 35\%

5. Nach Wiest und Walker: Peel No. 2 deep (Fa. Dermasence,

Glykolsäure 50\% und Salicylsäure 25\%) und TCA 35\%

Die Wirksamkeit und Eindringtiefe konnte histologisch bis in die papilläre Dermis nachgewiesen werden [7]. Indikation des mitteltiefen TCA-Peels ist die moderate Faltentherapie und solare Elastose. Die Behandlung perioraler Falten hat enttäuscht.

\section{Tiefes chemisches Peeling}

Zur Behandlung tiefer Falten, insbesonders perioraler Falten, liefert das Phenol-Peeling überzeugende Ergebnisse. Selbst tiefe Falten verschwinden bzw. werden gemildert ( $\bullet$ Abb. 18). Ein sichtbarer Straffungseffekt tritt zusätzlich ein. Außerdem werden Hautstruktur und -textur wesentlich verbessert. Auch das fahle Hautkolorit verschwindet. Die Eindringtiefe beträgt ca. 0,6 mm und reicht bis in die retikuläre Dermis. Schon 1952 wurden am Meerschweinchen histologische Untersuchungen nach PhenolPeels durchgeführt und kompakte Kollagenbündel wurden in paralleler Anordnung in der oberen Dermis nachgewiesen [2]. Diese Ergebnisse wurden 1960 durch Ayres bestätigt. Auch hier wurden neue Kollagenfaserbündel subepidermal in paralleler Anordnung nachgewiesen [8]. Weitere Untersuchungen konnten diese Ergebnisse bestätigen. Interessanterweise steigt die Schädigung der Haut mit Erhöhung der Konzentration des Phenols nicht an. $100 \%$-Phenol-Lösung zerstört die Dermis nur unwesentlich (histologisch bestätigt) mehr als 25\%ige Lösung [9]. Histologische Untersuchungen nach Phenol-Peeling wurden durchgeführt. So hat Baker Biopsien von 22 Patienten gewonnen, die sich einem Phenol-Peel unterzogen haben. Hier war der Nachbeobachtungszeitraum bis 13 Jahre nach durchgeführtem Peeling. Er konnte histologisch eine verminderte solare Elastose sowie eine deutliche Vermehrung elastischer Fasernetze nachweisen. Dies korreliert mit dem lang anhaltenden klinischen Effekt des Phenol-Peelings [10]. Auch Kligman et al. gewannen Biopsien aus Phenol behandelter Haut 1,5 bis 20 Jahre nach dem Peeling und konnten diese Ergebnisse bestätigen. Die Melanozyten werden nicht durch ein Phenol-Peeling eliminiert und waren sogar vereinzelt vermehrt. Die Melaninsynthese war unvollständig, Melaningranula waren epidermal nachweisbar. Somit erklärt sich die Hypopigmentierung, eine Depigmentierung findet nach einem Phenol-Peel nicht statt [11]. Unsere klinischen Erfahrungen bestäti- 

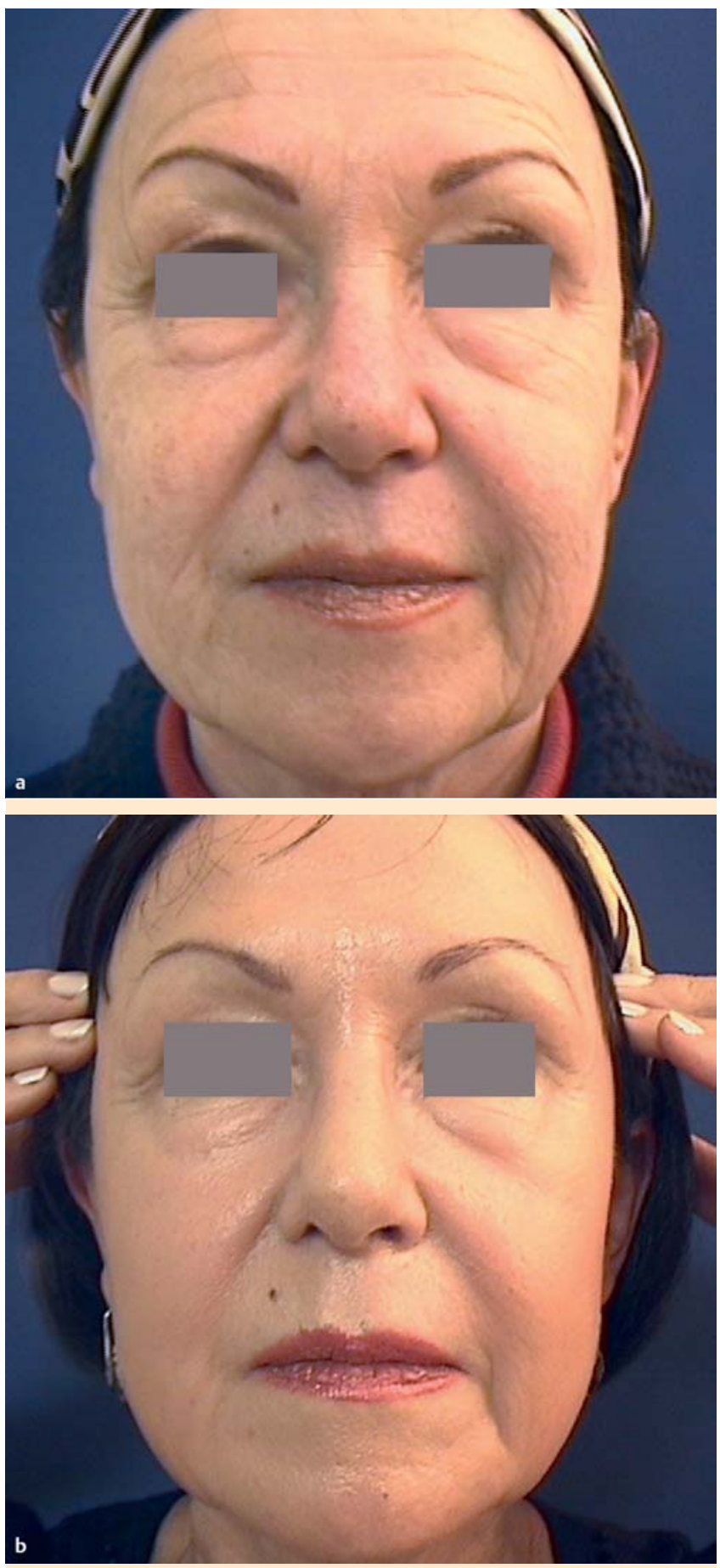

Abb. 1 a Vorher. b 6 Monate post Baker-Gordon-Peel.

gen das (siehe Text histologische Untersuchung). Die Haut wird nach einem Phenol-Peeling heller und frischer ( $\bullet$ Abb.1-3),

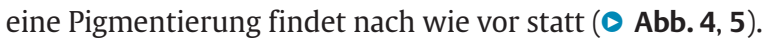

\section{Okklusion}

Im Gegensatz zu einem Trichloressigsäure-Peeling sollte nach dem Auftragen der Phenol-Lösung eine Okklusion erfolgen. Dieses kann entweder mit einer Vaseline-Creme oder besser aber mit einem Tape-Verband erfolgen. Der Okklusionsverband erhöht die Penetration des Phenols und verbessert somit die klinischen Ergebnisse. Dieses konnten Spira [9] und Stegman [5,6] histologisch nachweisen.
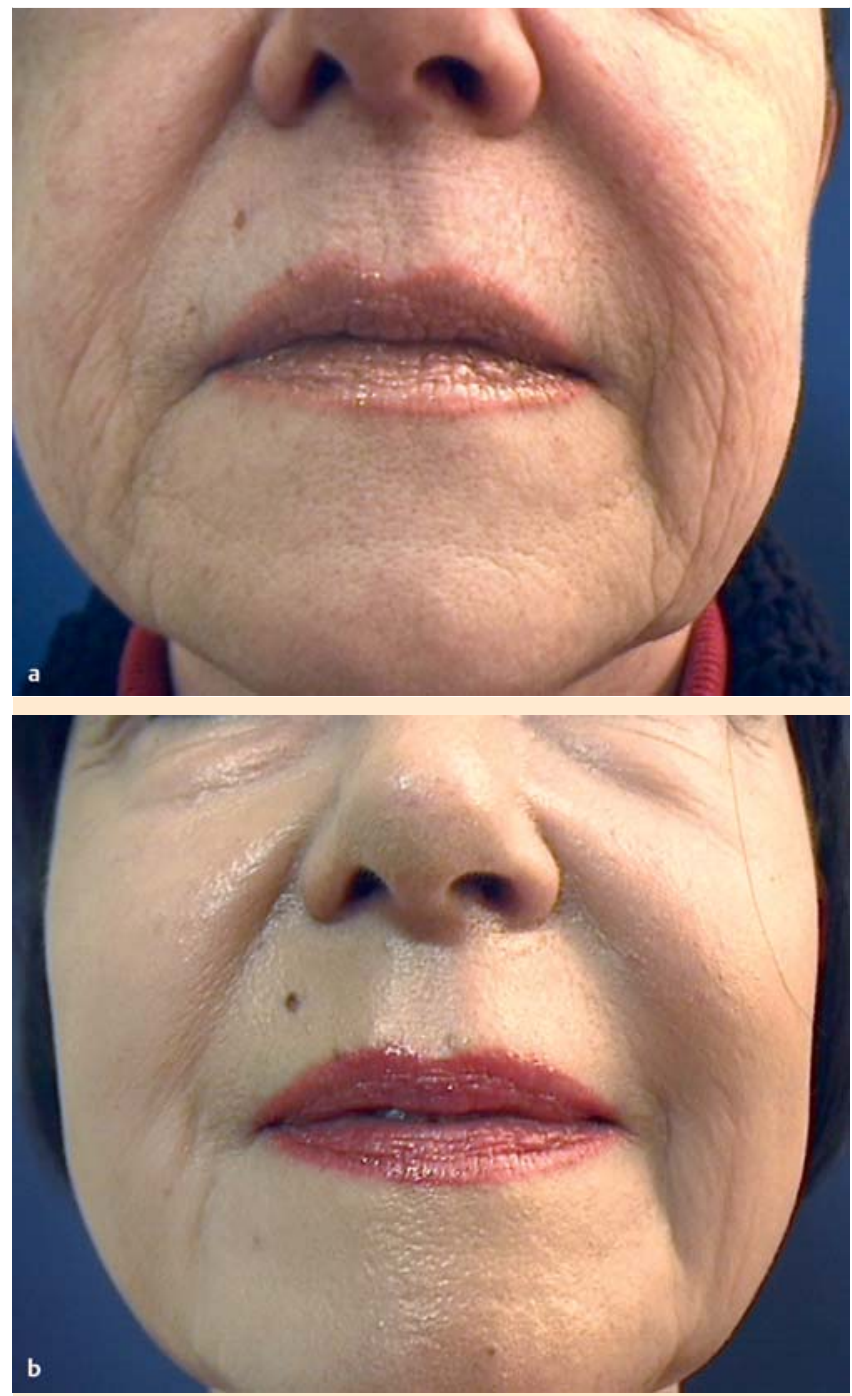

Abb. 2 a Vorher: Detailaufnahme. b 6 Monate post Baker-Gordon-Peel.

\section{Durchführung des chemischen Peeling mit Phenol $\nabla$}

\section{Allgemeines}

Chemisch handelt es sich bei Phenol um eine Karbolsäure, die 1834 durch Ferdinand Runge entdeckt wurde. In der Konzentration von $1 \%$ ist Phenol bakteriostatisch, über $1 \%$ sogar bakterizid. Phenol besitzt eine lokalanästhetische Wirkung. Gelöstes Phenol besteht aus Phenol 88\% in Wasser. Es ist fettlöslich und kann durch Auftragen von Glycerin von der Haut entfernt werden. Phenol wird schnell perkutan aufgenommen. Daher müssen PhenolPeelingmaßnahmen langsam durchgeführt werden, um die Resorption und die damit möglichen Nebenwirkungen gering zu halten. 88\%iges auf die Haut aufgetragenes Phenol führt zur sofortigen Keratokoagulation und damit zum Stopp der Eindringtiefe. Daher ist reines Phenol weniger effektiv. Die klassische Phenol-Formel ist die nach Baker-Gordon ( Tab. 2). Hierbei liegt die Phenol-Konzentration bei ca. 50-55\% und die Eindringtiefe reicht bis in die retikuläre Dermis [12]. Verwendet man hingegen Phenol-Lösungen in einer Konzentration von 30\% oder weniger, so ist der Effekt geringer. Wir verwenden diese nach Hetter modifizierten Lösungen zum Peeling des Halses mit o.a. Lösung ( Tab.2). Hexachlorophen wird als Oberflächenspannungherabsetzendes Agens zugegeben, um ein gleichmäßigeres Peeling 


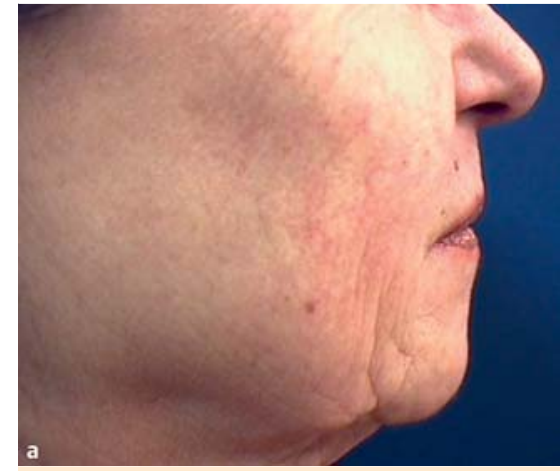

Abb. 3 Detail-

aufnahme.

a vorher, b 6 Monate post Peel.

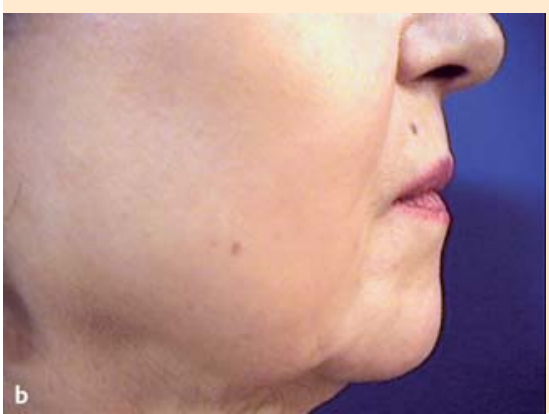

zu ermöglichen. Das aus dem Nachtschattengewächs Croton tiglium gewonnene Öl dient als epidermolytisches Vesikans, das die Wirkung des Phenols verstärkt und zu einer deutlichen Entzündung und zur Kollagenneogenese führt (siehe Abschnitt Rolle des Crotonöls). Die Lösung mischt sich nicht und muss permanent gerührt werden. Eine längere Lagerung ist nicht empfehlenswert. Eine ca. ein Jahr alte Lösung zeigte einen deutlichen Wirkverlust gemäß unserer Erfahrung. Wir verwenden seither nur frisch hergestellte Baker-Gordon-Peel-Lösung.

\section{Technik}

Das chemische Peeling bedarf eines gewissen manuellen Geschickes. Zunächst wird das Gesicht desinfiziert und gründlich mit Aceton entfettet. Generell kann durch den Applikationsdruck und die Anzahl der Applikationen die Eindringtiefe variiert werden mit unterschiedlichen Konsequenzen. Im Gegensatz zur Lasertherapie, bei der technisch die Eindringtiefe festgelegt ist, kann diese beim Peel sehr schnell variieren. Als einzigen klinischen Parameter zur Bestimmung der Eindringtiefe dient das so genannte „frosting“. Es beschreibt die mehr oder weniger schnell eintretende Weißfärbung der Haut (siehe $\bullet$ Abb.6). Es handelt sich hier um eine Keratokoagulation, das heißt epidermales und dermales Eiweiß fällt aus. Dieses stoppt das weitere Eindringen der Säure. Die Eindringtiefe des Phenol-Peels reicht bis in die retikuläre Dermis. Die Lösung wird unter permanentem Rühren langsam und gleichmäßig auf die Haut mit einem Watteträger aufgetragen. Das Frosting sollte weißlich-gräulich sein. Augenbrauen und das Lippenrot werden mitgepeelt. Auch die Augenoberlider werden bis zum Tarsus behandelt ( $\bullet$ Abb. 7), allerdings weniger kräftig. Mit einem Verlust von Haaren oder auch permanentem Make-up ist nicht zu rechnen. Starke Falten werden mit einem mit Watte umwickelten Zahnstocher nachgearbeitet. Die Perioralregion kann wegen der Dicke der Dermis kräftiger gepeelt werden. Wichtig ist die Demarkationslinie zu verstecken und fließende Übergänge zu schaffen. Daher wird an der Stirn bis in den Haaransatz gepeelt, die Ohrläppchen werden mitbehandelt und die Demarkationslinie ist im Kieferwinkel und unterhalb des Kinnes. Im Anschluss an das Peel wird ein Okklusiv-
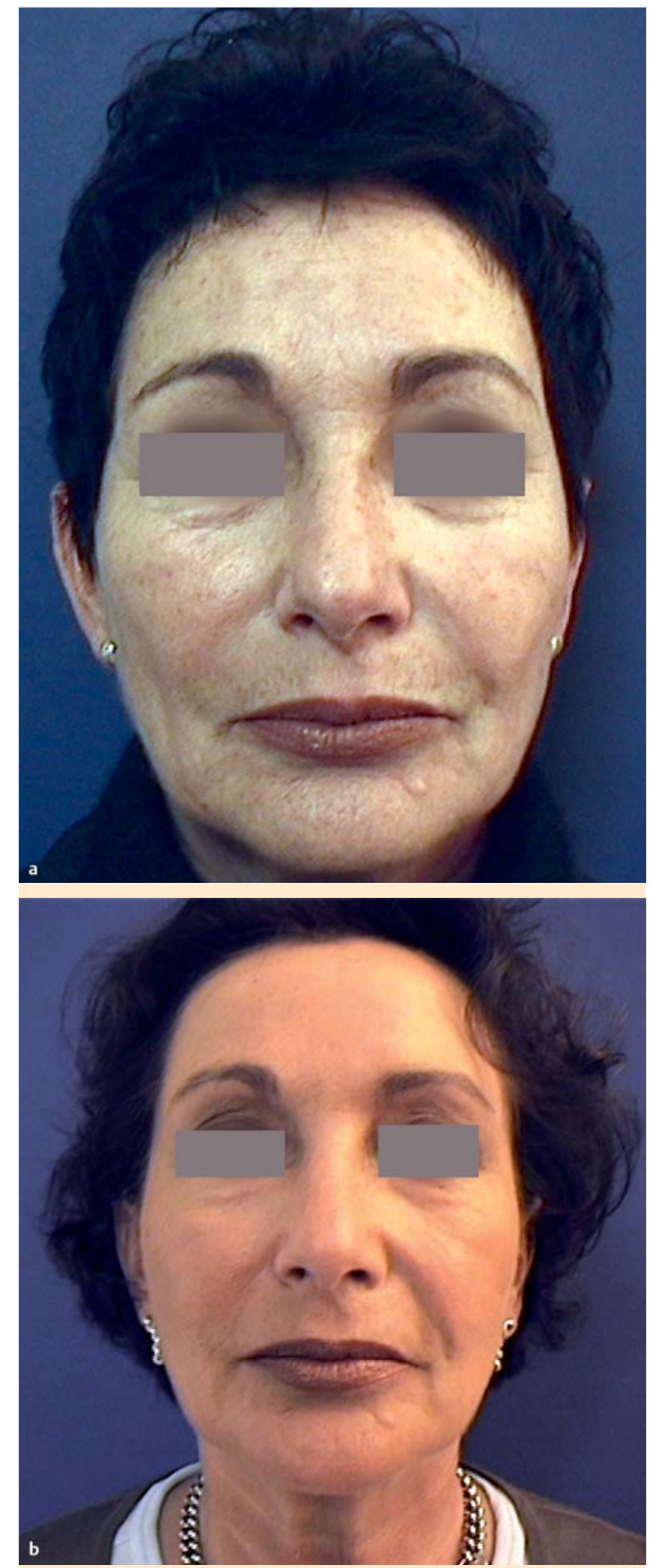

Abb. 4 Vor Phenol-Peel nach Baker-Gordon. b 2 Jahre post Peel mit gutem Ergebnis und erhaltener Pigmentierung.

verband für 24 Stunden direkt auf die Haut aufgeklebt ( $\bullet$ Abb. 8). Das Brennen verstärkt sich hierunter nochmals für die Dauer von ca. 8-9 Stunden. Die Schmerzhaftigkeit des Phenol-Peelings erfordert eine Sedo-Analgesie. Auch wegen der möglichen Arrhythmien ist die Anwesenheit eines Anästhesisten sinnvoll. In eige- 


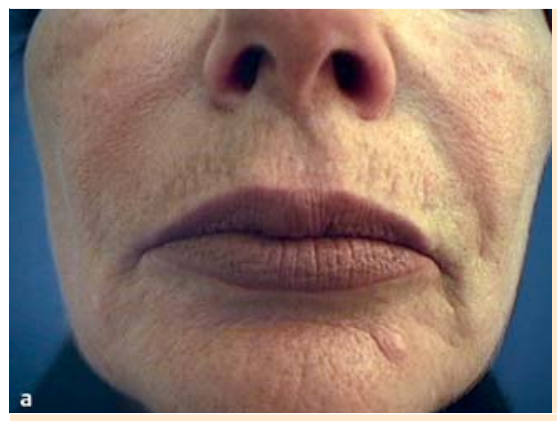

Abb. 5 Detailaufnahme.
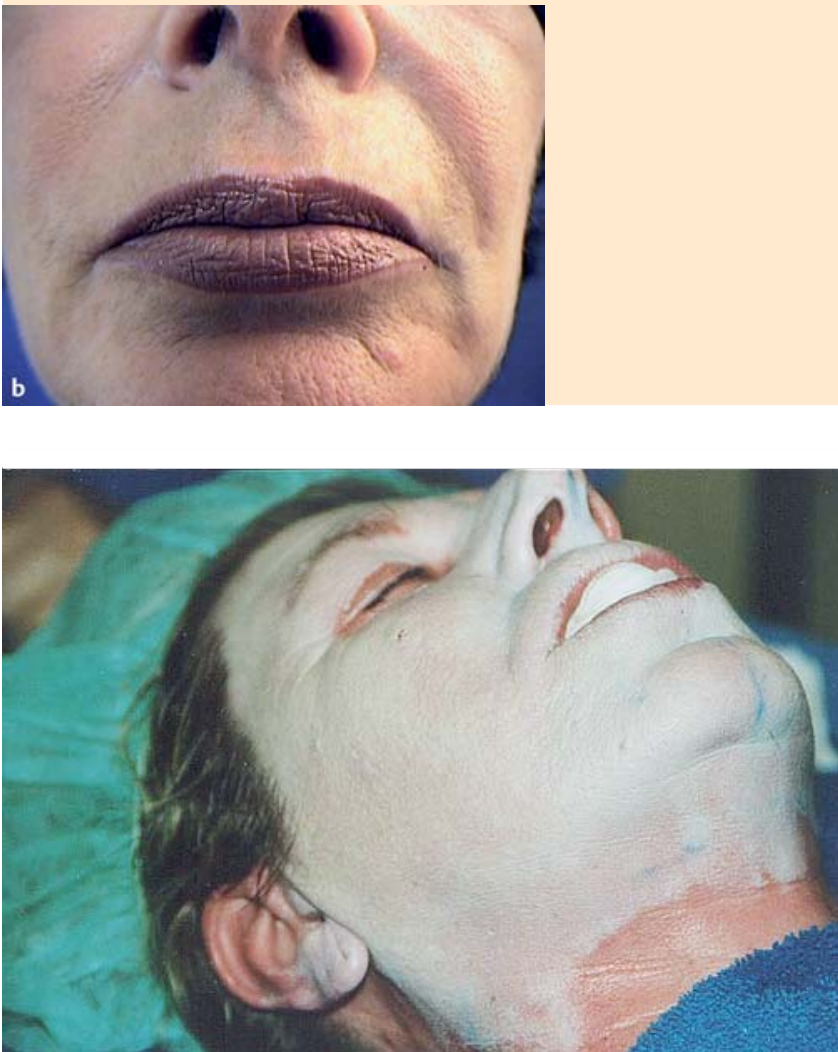

Abb. 6 Frost-Grad III.

Tab. 2 Rezeptur der Peel-Lösung.

\begin{tabular}{|c|c|c|c|}
\hline \multicolumn{2}{|c|}{ Baker-Gordon-Formel } & \multicolumn{2}{|c|}{$\begin{array}{l}\text { modifizierte Lösung } \\
\text { für Dekolleté }\end{array}$} \\
\hline Phenol USP $88 \%$ & $3 \mathrm{ml}$ & Phenol & $6,5746 \mathrm{~g}$ \\
\hline $\begin{array}{l}\text { destilliertes } \\
\text { Wasser }\end{array}$ & $2 \mathrm{ml}$ & $\begin{array}{l}\text { gereinigtes } \\
\text { Wasser }\end{array}$ & $13,6450 \mathrm{~g}$ \\
\hline Flüssigseife & 8 Tropfen & Triclosanum & $0,0009 \mathrm{~g}$ \\
\hline Crotonöl & 3 Tropfen & Crotonöl LGG & $0,0204 \mathrm{~g}$ \\
\hline
\end{tabular}

nen Versuchen, haben wir auch Phenol-Peels ohne Anästhesie nur mit einem Tranquilizer und Metamizol-Tropfen durchgeführt. Auch Kombinationspeeling-Behandlungen (Phenol in einigen Bereichen und TCA) können so durchgeführt werden. Das gesamte Peeling dauert ca. 1 Stunde ohne die Vorbereitungszeit. Danach sollte auf eine ausreichende Flüssigkeitszufuhr geachtet werden. Eine systemische Antibiose ist nicht erforderlich. Eine Herpesprophylaxe unumgänglich.

Eine spezifische Vorbehandlung ist umstritten. In einem klinischen Halbseitenversuch haben wir eine Gesichtshälfte mit einer

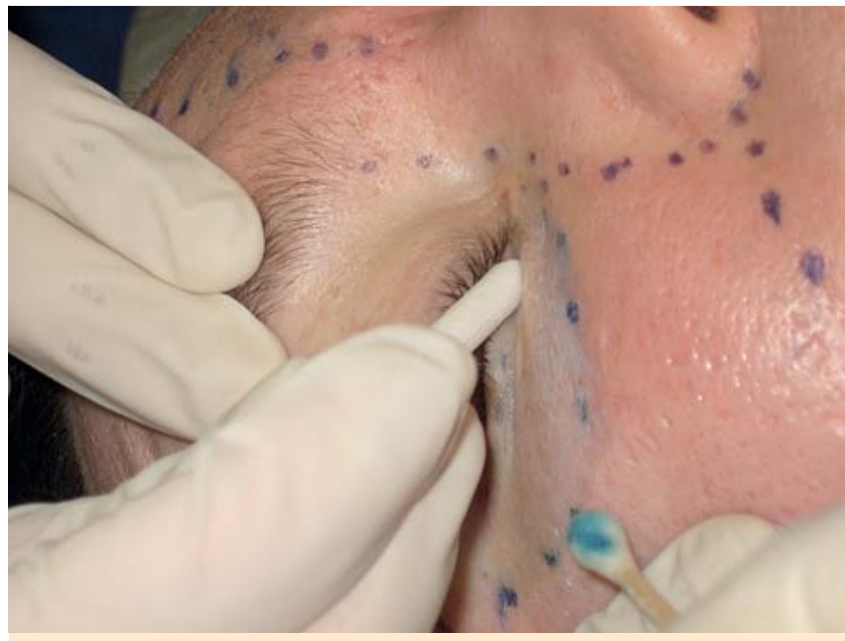

Abb. 7 Auftragen der Peel-Lösung am Auge.

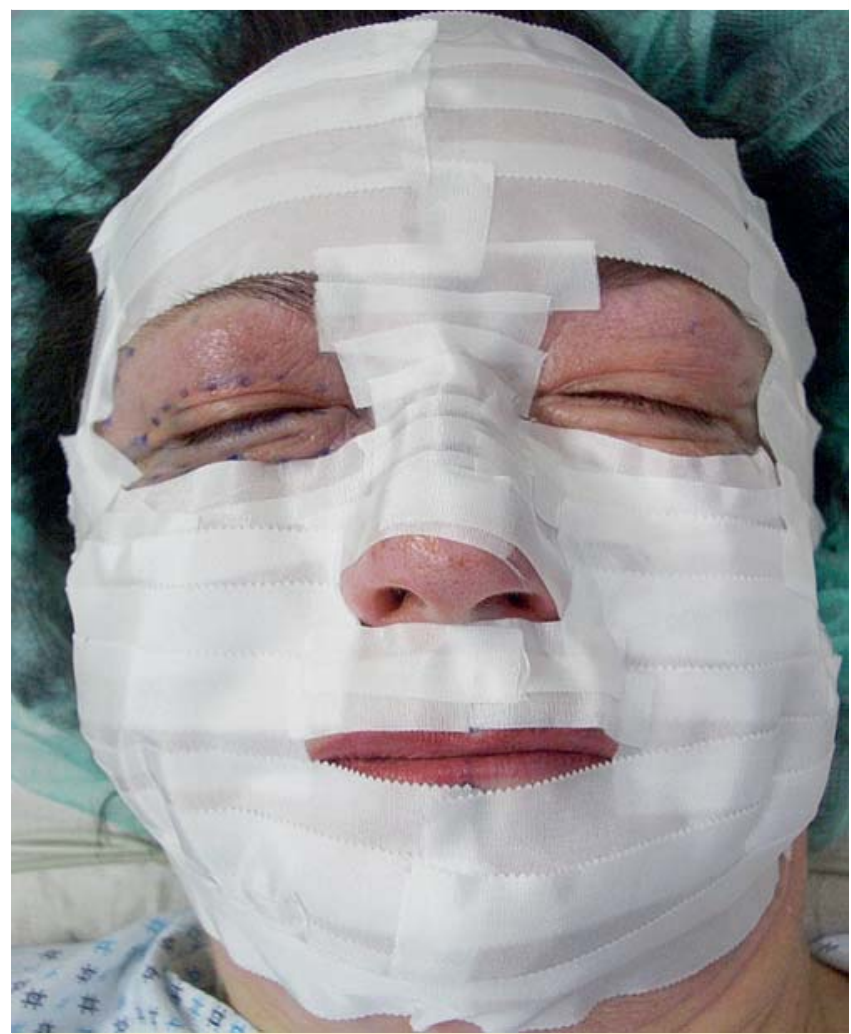

Abb. 8 Okklusiv-Verband unmittelbar post Peel.

Tretinoin- und Hydrochinon-haltigen Creme einseitig vorbehandeln lassen. An der vorbehandelten Seite war das Frosting gleichmäßiger und erschien schneller, über die klinische Relevanz kann noch keine Aussage getroffen werden. Unabdingbar ist ebenfalls eine Vorbehandlung mit Botulinumtoxin A in hohen Dosierungen, um die Mimik ruhigzustellen. Es hat sich gezeigt, dass es zu einem wesentlich besseren Kollagenaufbau kommt (Wiest, nicht publiziert, [13]). Durch die Ruhigstellung der muskulären Aktivität während der Wundheilungsphase nach Phenol-Peeling kann sich nicht wieder das alte Faltenmuster ausbilden. Durch die Ruhigstellung der Muskulatur mit Botulinumtoxin A verändert sich die Stoffwechsellage der Muskulatur, positive Effekte auf die Wundheilung wären denkbar. Wir applizieren Botulinumtoxin A zwei Wochen vor der Durchführung des Phenol-Peelings, kürzere 


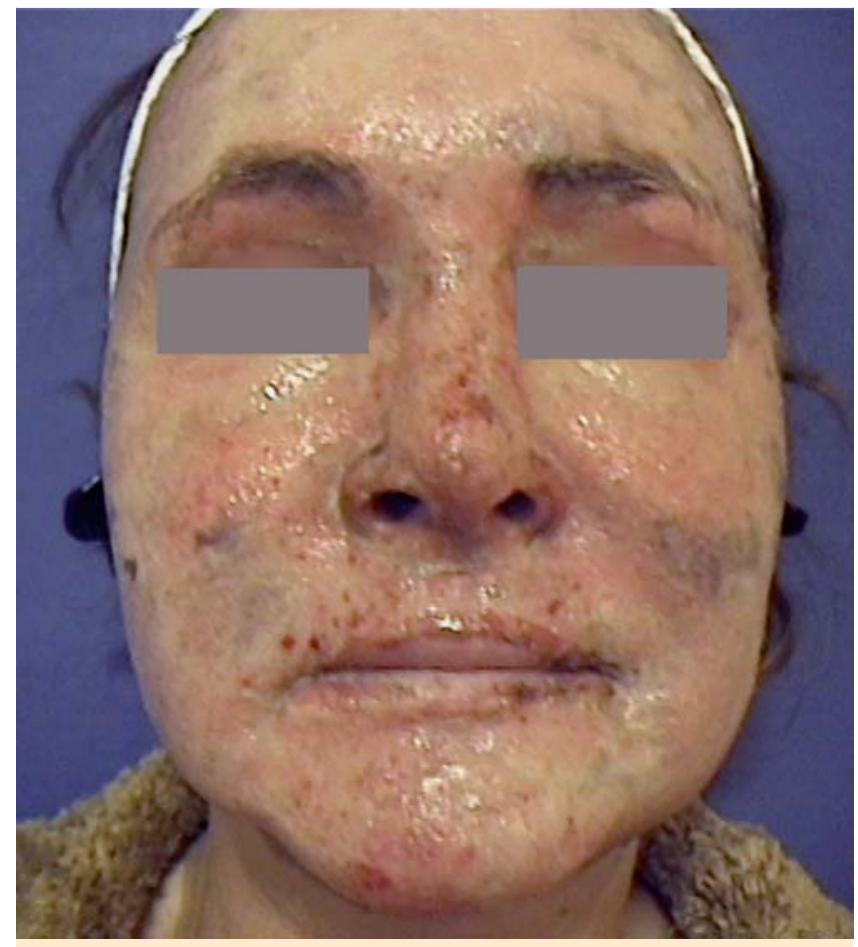

Abb.9 Unmittelbar nach Abnahme des Tape-Verbandes.

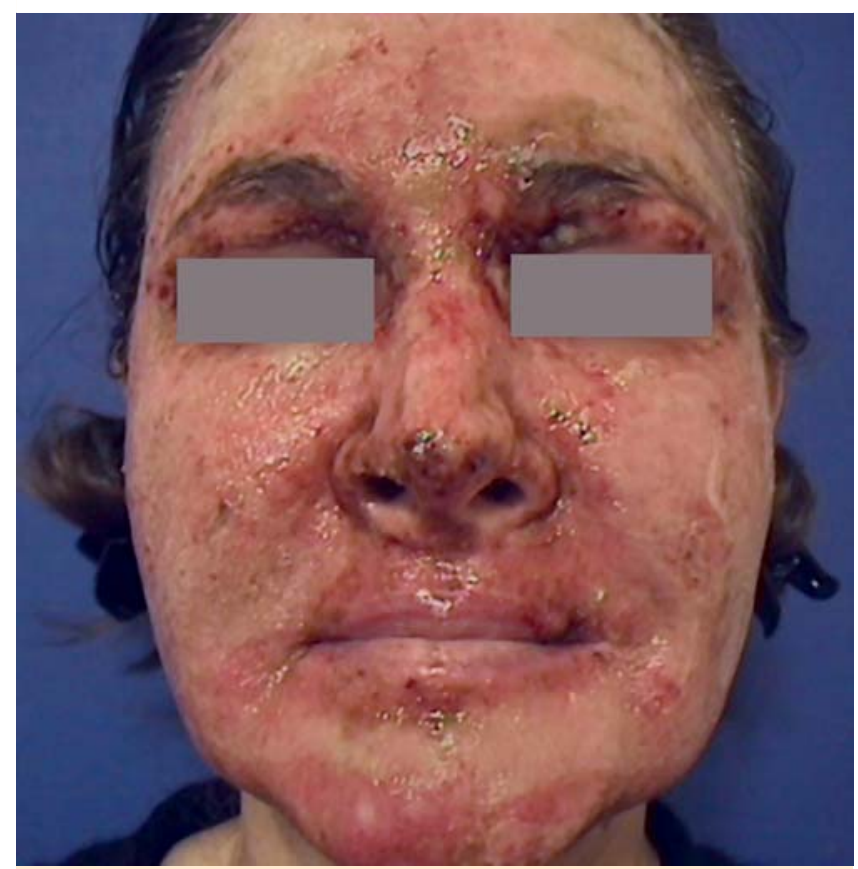

Abb. 1024 Stunden nach Abnahme des Verbandes.

Abstände sind wegen der Diffusionsgefahr nach eigenen Beobachtungen problematisch, weil es durch das massive Ödem des Phenol-Peels zu möglichen unerwünschten Nebenwirkungen kommen kann.

\section{Nachsorge}

Die postoperative Nachsorge ist aufwendig und erfolgt stadiengerecht ( Abb.9-11). Hier gibt es keine allgemein verbindlichen Regeln. Nach 24 Stunden wird der Tapeverband abgenommen, was in der Regel nicht schmerzhaft ist, da durch das Exsu-

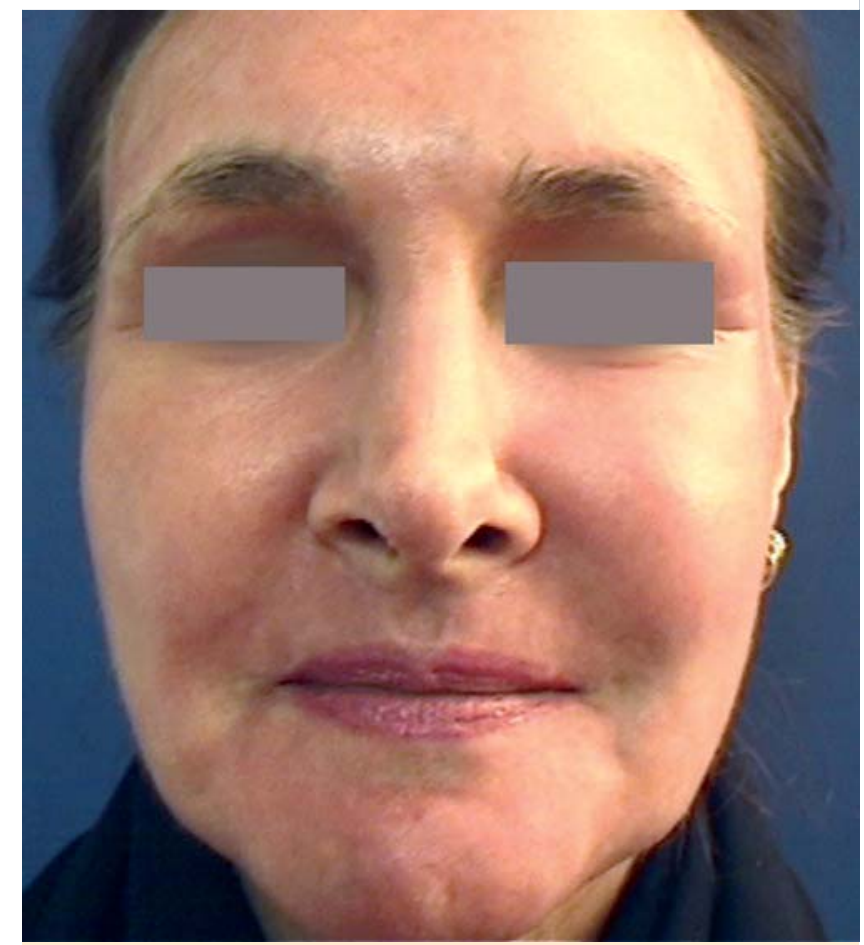

Abb. 1110 Tage post Peel.

dat der Verband fast von alleine „abschwimmt“. Die Wundversorgung besteht in täglichen Débridements und Anwendung von fett-feuchten Verbänden. Zur Förderung der Wundheilung verwenden wir nach dem vorsichtigen mechanischen Entfernen von Exsudat und Krusten mit einer Kompresse oder einer Pinzette ein hyaluronsäurehaltiges Serum. In der Regel fetten wir dann für 2-3 Tage mit Fusidinsäure-haltiger Salbe und Vaseline im Wechsel. Zwischendurch werden feuchte Kompressen aufgetragen. Vaseline wird gut toleriert, sollte aber wegen der verstärkten Milienbildung nicht länger als 10-14 Tage angewendet werden. Häufig tritt am 4. Tag ein Brennen und ein unangenehmes Hautgefühl auf. Dann sollte auf andere Produkte in Cremeform ausgewichen werden.

Nach 2-3 Tagen ist die Schwellung rückläufig, nach 10 Tagen sind die meisten Krusten abgeheilt und der Patient ist wieder gesellschaftsfähig. Die Rötung persistiert für ca. 2-4 Monate, kann aber in der Regel gut mit Make-up kaschiert werden. Die Therapie muss individuell und situationsgerecht erfolgen. Beschwerlich für den Patienten ist das Spannungsgefühl, welches ca. 2 Wochen anhält. Auch über eine länger persistierende verstärkte Schmerzempfindlichkeit einzelner Gesichtspartien, insbesondere der Temporalregion wird vermehrt berichtet. Das Débridement ist zeitintensiv. Der Patient wird über einen Zeitraum von 8 Tagen täglich in die Praxis einbestellt. Eine erfahrene Kosmetikerin oder geschulte Arzthelferin kann das Débridement und auch Lymphdrainagen durchführen. Eine ärztliche Kontrolle zur frühzeitigen Erkennung von möglichen Wundheilungsstörungen ist jedoch unabdingbar.

\section{Risiken und Nebenwirkungen}

Es handelt sich beim Phenol-Peeling um einen größeren, invasiven und aufwendigen Eingriff, der intensive Patientenvorbereitung und Aufklärung erfordert. Für den Patienten gestaltet sich die lange Ausfallszeit von 2 Wochen oft problematisch, ist aber unumgänglich. Das Risiko einer Narbenbildung ist gering und 

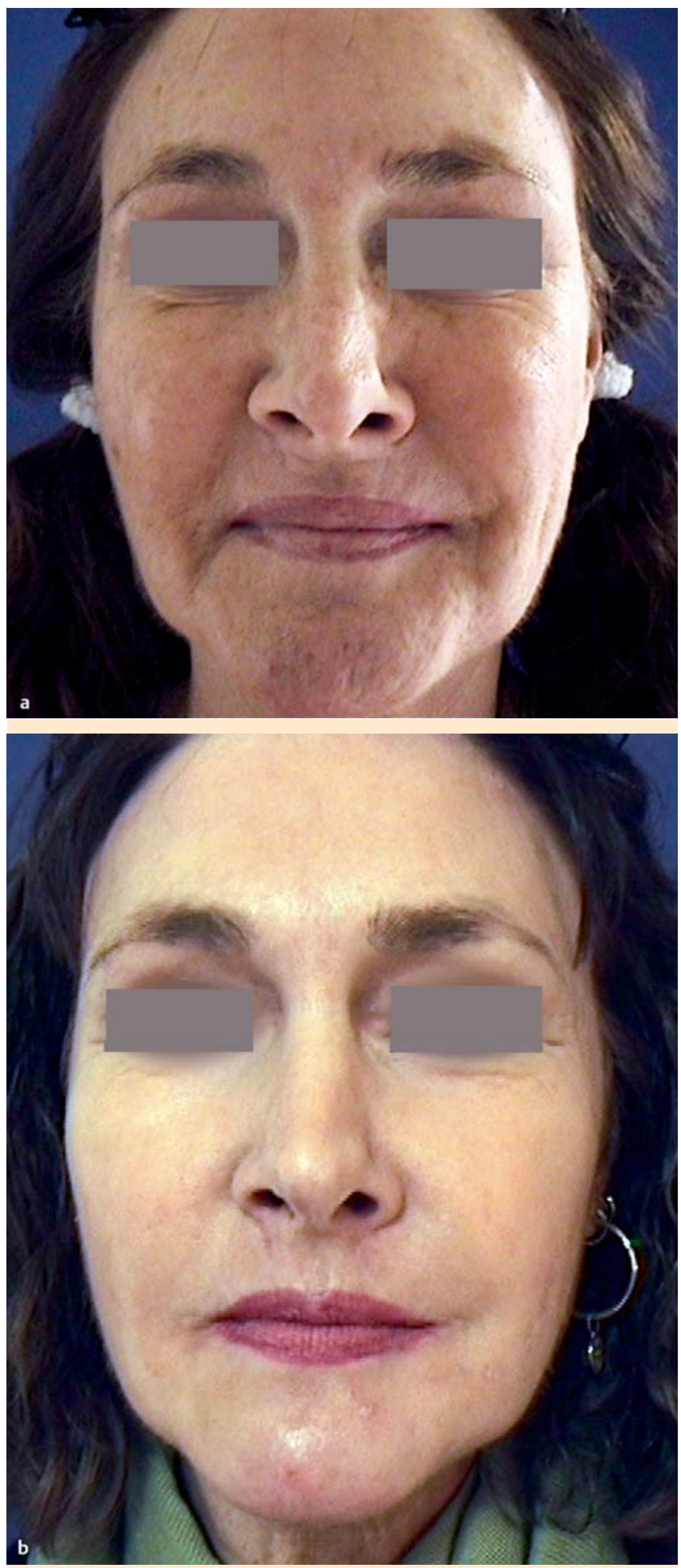

Abb. 12 Vor tiefem Phenol-Peel (ausgeprägte Lichtschädigung bei 48jähriger Patientin). b 2 Jahre post Peel.

kann mit $<1 \%$ angegeben werden, hängt aber vom Behandler ab. Die Risiken der Sedo-Analgesie sind bekannt und eher als gering einzustufen. Das Risiko der Arrhythmien kann durch das langsame Auftragen der Lösung deutlich gemindert werden. Nach neuen Publikationen wird das Risiko auf 6,6\% geschätzt. Allerdings sind alle kardialen Veränderungen dokumentiert worden, auch die, die klinisch nicht relevant waren (1/3 der dokumentierten Fälle). Insgesamt wird die Kardiotoxizität nach Phenol-Peel als

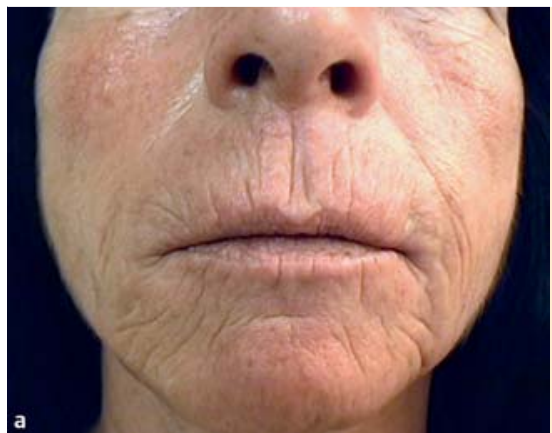

Abb. 13 Vor tiefem Baker-Gordon-Peel. $\mathbf{b}$ diskrete Unregelmäßigkeit in der Pigmentierung 10 Monate post Peel.

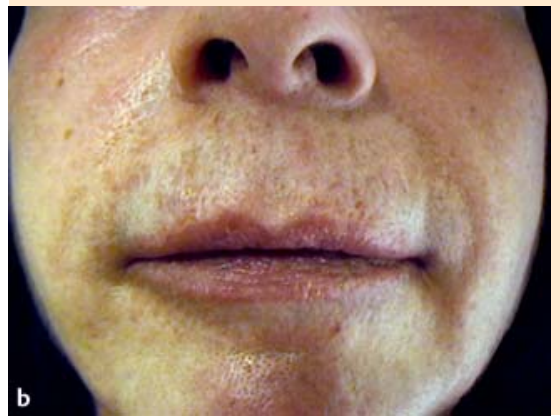

gering angegeben. Diabetes, Hypertension und Depression gingen mit einem erhöhten Arrhythmierisiko einher [14].

Die dem Phenol-Peel oft angelastete maskenhaft wirkende „Alabasterhaut" tritt nur nach mehrfach durchgeführten Peelingbehandlungen mit Phenol auf. Einmaliges Phenol-Peeling führt zu einer deutlichen Hautauffrischung, der Teint bekommt, wie eingangs erwähnt, seine rosige und helle Nuance zurück ( Abb. 12a,b). Auch Hauttyp III und IV nach Fitzpatrick kann behandelt werden, allerdings kann es zu diskreten unregelmäßigen Pigmentverschiebungen kommen ( $\mathrm{Abb}$. 13a,b).

\section{Histologische Veränderungen nach Phenol-Peel (0 Abb. 14-17)}

Die feingewebliche Untersuchung aktinisch geschädigter Haut vor Durchführung eines Phenol-Peels zeigt unter einer regelrecht stratifizierten Epidermis und einer weitgehend unauffälligen papillären Dermis eine von aktinischer Elastose durchsetzte retikuläre Dermis ( Abb. 14a, 15a, 16a). Typischerweise ist die aktinische Elastose im oberen Drittel der retikulären Dermis am ausgeprägtesten, setzt sich aber bis in die tiefe retikuläre Dermis fort. Das Ausmaß der aktinischen Elastose ist abhängig von der Lokalisation, so findet sich z. B. in der Haut der Wangenregion deutlich mehr aktinische Elastose als am seitlichen Hals ( $\bullet$ Abb. 14 d, 15d).

In 3 Monate nach Phenol-Peel entnommenen Gewebeproben ist die Epidermis regelrecht stratifiziert, zeigt jedoch ein abgeflachtes Reteleistenrelief ( $\bullet$ Abb. 14b, 15b). Die papilläre Dermis ist nicht abgrenzbar. In der retikulären Dermis finden sich horizontal parallel ausgerichtete kompakte Kollagenfasern durchsetzt von Fibroblasten ( $\bullet$ Abb. 15b). Das kollagene Bindegewebe dieser superfiziellen narbigen Fibrose ersetzt weitgehend die aktinische Elastose im oberen Drittel der retikulären Dermis. In der Elastika-Färbung ist der Verlust der aktinischen Elastose im oberen Drittel der retikulären Dermis sehr deutlich darstellbar ( Abb. 16b).Zusätzlich finden sich schüttere lymphohistiozytäre Infiltrate oberflächlich perivaskulär ( Abb. 14b, 15b). 


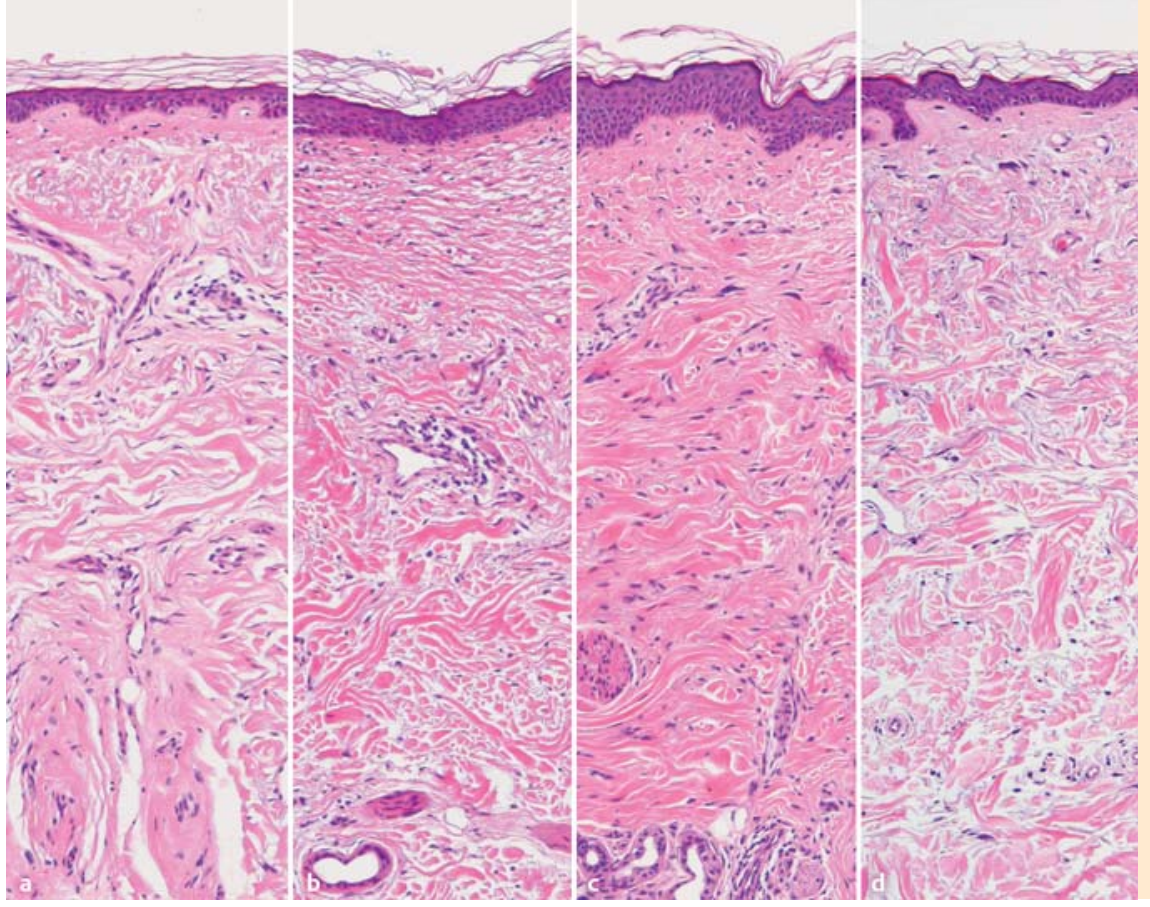

Abb. 14 a-d Biopsien aus der Wangenregion vor Phenolpeel (a), 3 Monate nach Phenol-Peel (b), 6 Monate nach Phenol-Peel (c) sowie unbehandelte Haut vom seitlichen Hals zeitgleich entnommen (d). Vor Phenol-Peel (a) sowie in der unbehandelten Haut (d) zeigt sich eine aktinische Elastose insbesondere im oberen Drittel der retikulären Dermis während 3 Monate und 6 Monate nach Phenol-Peel eine deutliche narbige Fibrose im oberen Drittel der retikulären Dermis die aktinische Elastose ersetzt hat. Hämatoxylin \& Eosin $\times 100$
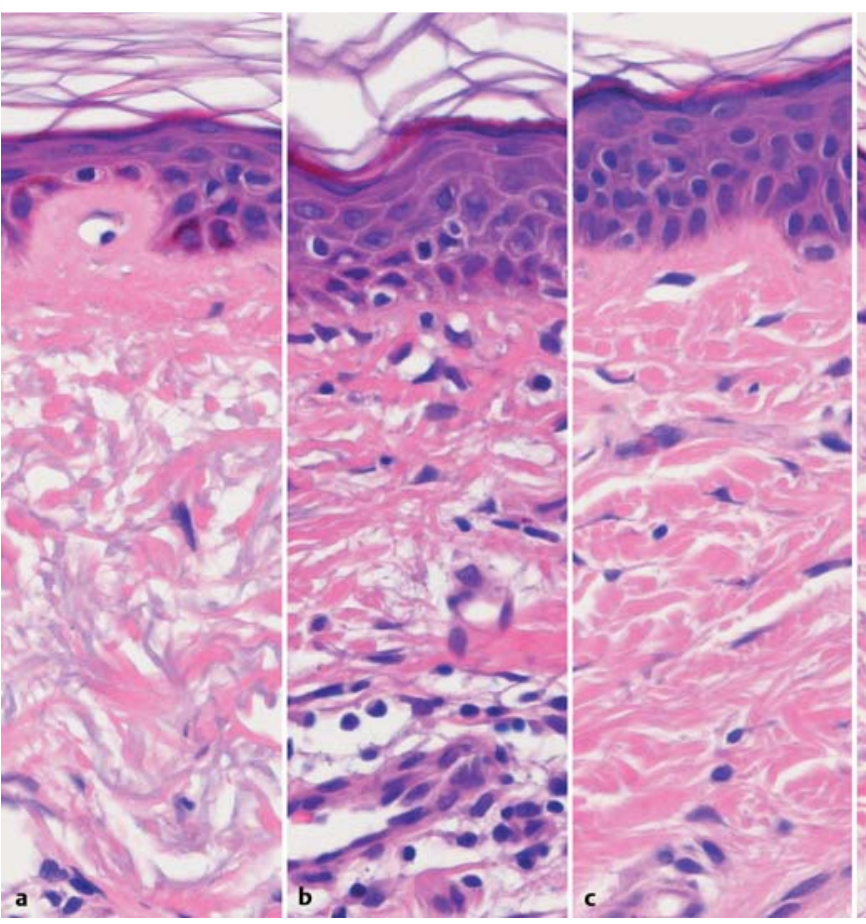

Sechs Monate nach Durchführung eines Phenol-Peels ist die superfizielle narbige Fibrose in der Dermis histologisch immer noch deutlich sichtbar, der Verlust der aktinischen Elastose kann weiterhin in der Elastika-Färbung herausgestellt werden ( $\triangle$ Abb. 14c, 15c, 16c). Allerdings ist die strukturelle Organisation des neu gebildeten kollagenen Bindegewebes verbessert: Kollagenfasern sind nicht mehr horizontal parallel orientiert, sondern beginnen sich netzartig auszurichten, ähnlicher einer normalen, nicht aktinisch geschädigten retikulären Dermis ( $\bullet$ Abb. 15c). Die papilläre Dermis und das epidermale Reteleistenrelief beginnen sich zu rekonstituieren. Ein Entzündungszellinfiltrat findet sich nicht mehr, jedoch sind die dermalen Fibroblasten weiterhin etwas vermehrt.

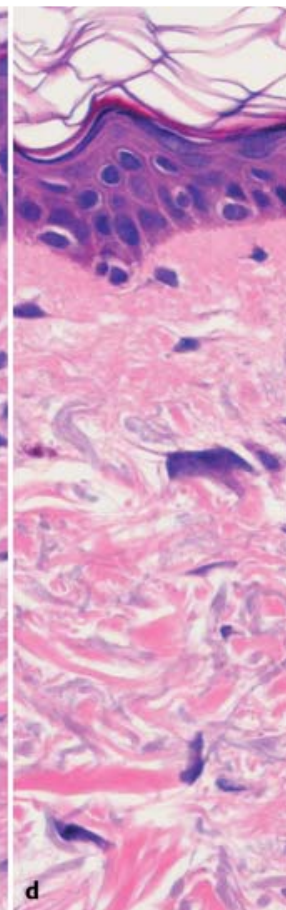

Abb. 15 a-d Biopsien aus der Wangenregion vor Phenolpeel (a), 3 Monate nach Phenol-Peel (b), 6 Monate nach Phenol-Peel (c) sowie unbehandelte Haut vom seitlichen Hals zeitgleich entnommen (d). Vor Phenol-Peel (a) sowie in der unbehandelten Haut (d) zeigt sich eine erhaltene papilläre Dermis während 3 Monate nach Phenol-Peel (b) die papilläre Dermis nicht darstellbar ist, die epidermalen Reteleisten sind verstrichen. Subepidermal finden sich parallel horizontal ausgerichtete Kollagenfasern und etwas vermehrte Fibroblasten sowie perivaskuläre Entzündungszellinfiltrate. Sechs Monate nach Phenol-Peel ist die narbige Fibrose besser organisiert mit retikulär verwobenen Kollagenfasern und ohne Entzündungszellinfiltrate (c), die papilläre Dermis ist flach rekonstituiert. Hämatoxylin \& Eosin $\times 200$.

Neben den strukturellen Veränderungen in der retikulären Dermis fällt infolge der Phenol-Behandlung auch eine geringe Erhöhung der Melanozytendichte in der basalen Epidermis auf ( $\bullet$ Abb.17a-d). Ähnlich der Situation in der Epidermis nahe von frischen Operationsnarben, kann in der Phenol-behandelten Haut eine Zunahme der Anzahl der basalen Melanozyten festgestellt werden ( $\bullet$ Abb.17b), die auch bis zu 6 Monate nach Phenol-Behandlung noch anhält ( $\bullet$ Abb. 17d).

Zusammenfassend kann festgestellt werden, dass die histologischen Veränderungen im Sinne einer superfiziellen Fibrose mit Verdrängung der vorbestehenden aktinischen Elastose einen klinisch deutlich sichtbaren Glättungseffekt („rejuvenation“) an der Haut erklären können. 

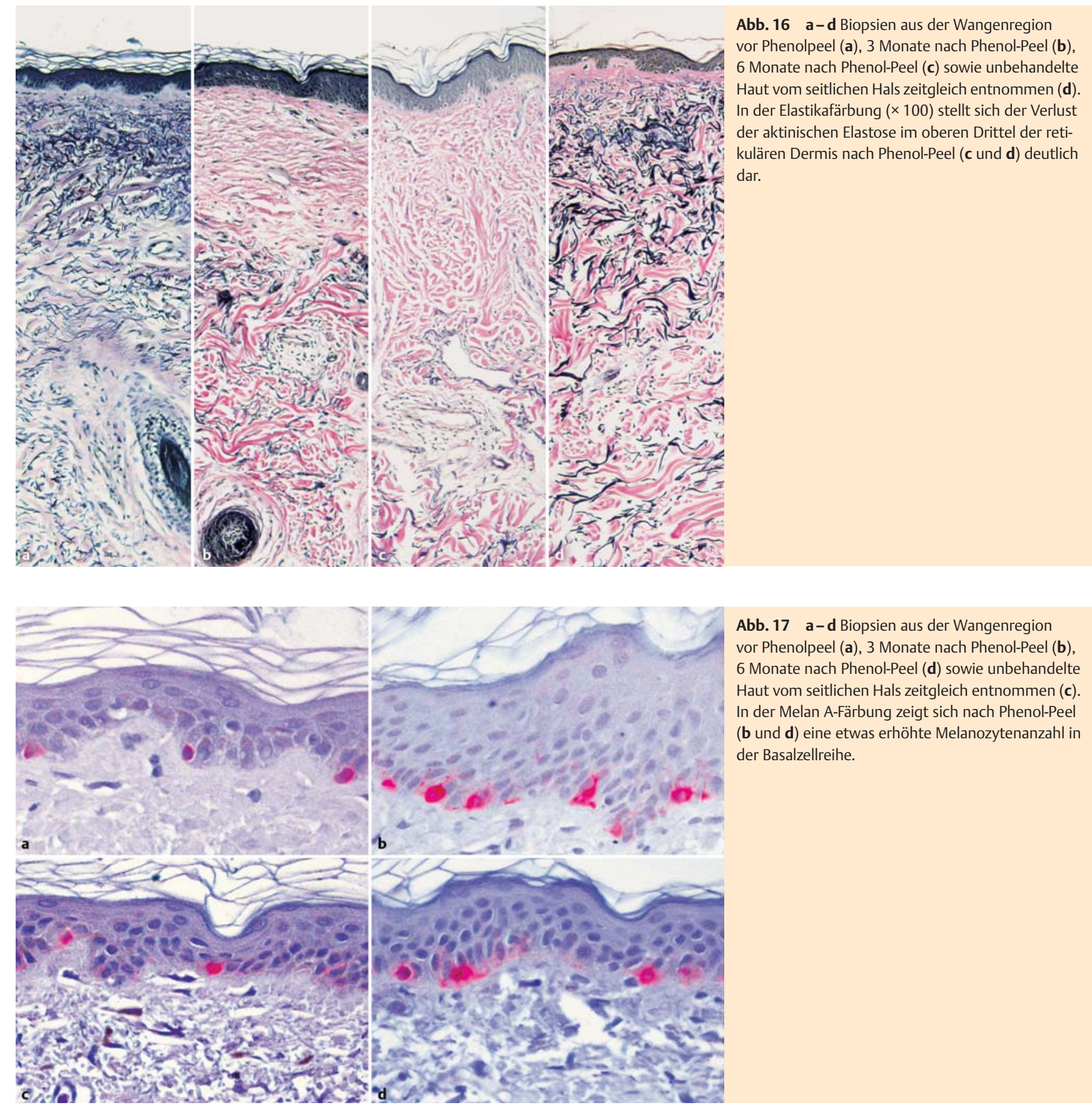

Abb. 17 a-d Biopsien aus der Wangenregion vor Phenolpeel (a), 3 Monate nach Phenol-Peel (b), 6 Monate nach Phenol-Peel (d) sowie unbehandelte Haut vom seitlichen Hals zeitgleich entnommen (c). In der Melan A-Färbung zeigt sich nach Phenol-Peel (b und d) eine etwas erhöhte Melanozytenanzahl in der Basalzellreihe.

\section{Renaissance?}

Die Vorteile des chemischen Peelings mit Phenol sind klar: Die Verbesserung des Hautbildes ist deutlich - bei geringem technischem Aufwand. In Halbseitenstudien konnte die Überlegenheit des Phenol-Peeling gegenüber dem $\mathrm{CO}_{2}$-Laser nachgewiesen werden $[15,16]$. Außerdem sind die Ergebnisse lang anhaltend und mehrere Zeichen der Hautalterung werden gleichzeitig therapiert. Die Studienlage zu Laser Skin Rejuvenation ist eher schlecht. Auch problematische Falten, wie die periorale Faltenbildung, können angegangen werden ( $\bullet$ Abb. 18a,b). Diese sind selbst einem Face-lift nicht zugänglich. Das Verfahren des Phenol-Peelings wird seit über 100 Jahren durchgeführt. Entsprechende histologische Untersuchungen sind durchgeführt worden und die Wirksamkeit konnte vielfach bestätigt werden $[2,3,5,6,9-12,15,16]$. Auch eigene histologische Untersuchungen haben die Wirksamkeit erneut bewiesen. Wir konnten in
Biopsien ( $\bullet$ Abb. 14-17) schon nach 6 Tagen die Kollagenneubildung nachweisen (Prof. Stolz, Hautklinik München Schwabing). Auch Verlaufsbiopsien konnten zwar die massive toxische Reaktion nachweisen, andererseits aber auch den schnellen Heilungsverlauf dokumentieren. Ein entscheidender weiterer Vorteil ist die Technologieunabhängigkeit des Verfahrens. Die Zusammensetzung des Phenol-Peels ist seit 1962 unverändert geblieben. Die Verbrauchsmaterialien sind kostengünstig.

Das Verfahren bietet somit entscheidende Vorteile: Sicher in der Anwendung, die Ergebnisse sind vorhersagbar, Non-Responder gibt es nicht. Dennoch ist der Informationsstand zum PhenolPeeling gering. Die Substanzen sind preiswert und über die Apotheke zu beziehen. Zum anderen sind die Substanzen negativ BGA-monographiert, was die rechtliche Situation erschwert. Dies gilt aber auch für Botulinumtoxin, sobald man es nicht für die Glabella-Falte verwendet. Sicher liegt ein Teil der Zurückhal- 


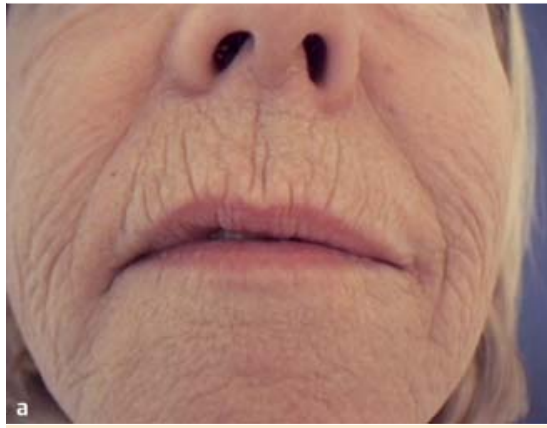

Abb. 18 65-jährige Patientin vor Baker-Gordon-Peel. b 2,5 Jahre post Peel.

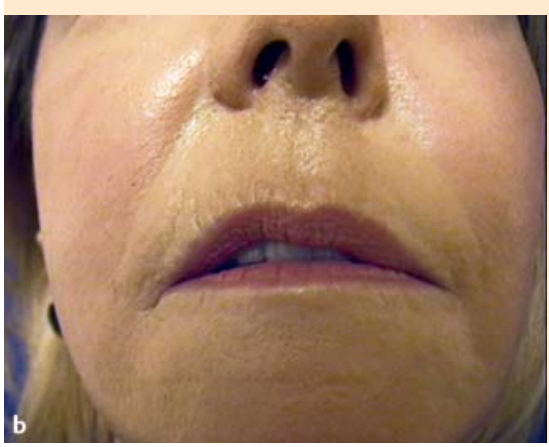

tung in der bekanntlich starken Schwellung, der massiven Exsudation und Krustenbildung sowie der aufwendigen Nachsorge begründet. Beim Phenol-Peel handelt es sich um ärztliche Kunst im ureigenen Sinn: eine gute Ausbildung, Übung, fundiertes Wissen, ein bisschen Mut, manuelles Geschick , straffe aber dennoch einfühlsame Patientenführung, und vor allem Zeit ... sind unerlässlich für ein gutes Gelingen.

\section{Abstract}

\section{Deep Chemical Peeling with Phenole - Renaissance of a Gold Standard \\ $\nabla$}

Chemical peels have been well known for thousands of years. In the Papyrus Ebers the first chemical peels have been described. Deep chemical peels were performed by lay surgeons in the $40 \mathrm{~s}$ in the US. First clinical investigations and observations were done by Baker and Gordon in the early sixties and by that time chemical peels with phenol were medically accepted and well known in plastic surgery and one of the most used methods for skin rejuve- nation. With the development of new ablative laser systems the knowledge and experience of the deep peel got lost. As a matter of fact lasers are an important tool for skin rejuvenation, but sometimes overestimated and long-term effects of new systems are not known or proven. Many laser systems are on the market, whereas the excellent results promised still have to be proven. Deep chemical peeling with Phenol gives long lasting and excellent results. Patient's satisfaction is extremely high related to the cosmetic outcomes.

\section{Literatur}

1 Unna PG. Therapeutiques génerales des maladies de la peau. 1882

2 Mackee GM et al. The treatment of post acne scars with phenol. Br J Dermatol 1952; 64: 456-459

3 Baker TJ. Chemical face peeling and rhytidectomy. Plast Reconstr Surg 1962; 29: 199

4 Baker TJ, Gordon HL. The ablation of rhytides by chemical means: A preliminary report. J Fla Med Assoc 1961; 48: 541

5 Stegman SJ. A study of dermabrasion and chemical peels in an animal model. J Dermatol Surg Oncol 1980; 6: 490

6 Stegman SJ. A comparative histologic study of the effects of the three peeling agents and dermabrasion on normal and sundamaged skin. Aesthetic Plast Surg 1982; 6: 123-135

7 Tse $Y$ et al. „A clinic and histological evaluation of two medium depth peels. Glycolic acid versus Jessner's trichloroacetic acid“. Dermatol Surg 1996; 22: $781-786$

8 Ayres S. Dermal changes following application of chemical cauterants to aging skin. Arch Dermatol 1960; 82: 578 - 585

9 Spira $M$ et al. Chemosurgery: A histologic study plast. Reconstr Surg 1970; 45: 247

10 Baker TJ et al. Long-term histological study of skin after chemical face peeling. Plast Reconstr Surg 1974; 53: 522

11 Kligman AM, Baker TJ, Gordon HL. Long-term histologic follow up of phenol face peels. Plast Reconstr Surg 1985; 75: 652 - 659

12 Brown AM et al. Phenol induced histological skin changes: Hazards, techniques and uses. Br J Plast Surg 1960; 13: 158 - 169

13 Kanduc BV et al. „Botulinum toxin A adjuncitve use in manual chemabrasion: controlled long term study for treatment of upper perioral wrinkles“. Dermatol Surg 2007; 33: 066-1072

14 Landau M. Cardiac complicationsin deep chemical peels. Dermatol Surg 2007; 33: 190-193

15 Chew J et al. Treatment of upper lip wrinkels: a comparison of 950 microsec dwell time carbon dioxide laser with unoccluded Baker's Phenol chemical peel. Dermatol Surg 1999; 25: 262 - 266

16 Langsdorn PR et al. Comparison of the laser and phenol chemical peel in facial skin resurfacing. Arch Otolaryngol Head Neck Surg 2000; 126: $195-1199$

17 Matic $D B$ et al. The effect of botulinum toxin type A on muscle blood perfusion and metabolism. Plast Reconstr Surg 2007; 120: 1823-1833 